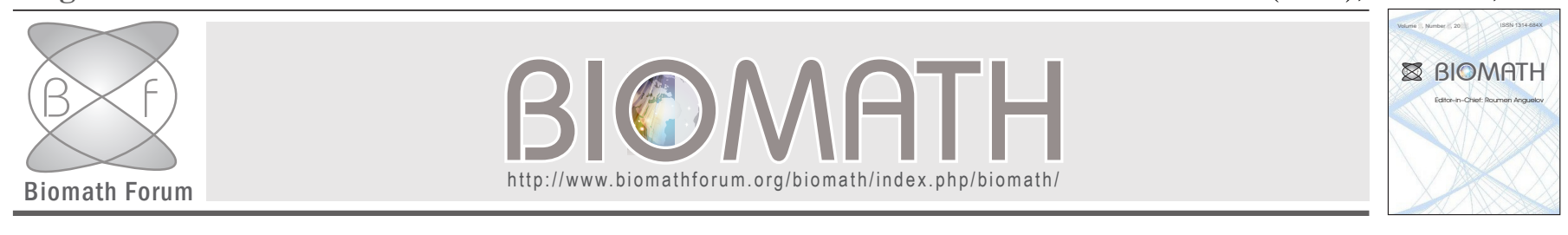

\title{
Multiregional SIR Model with Infection during Transportation
}

\author{
Diána H. Knipl* and Gergely Röst ${ }^{\dagger}$ \\ *Analysis and Stochastics Research Group - Hungarian Academy of Sciences, \\ Bolyai Institute, University of Szeged, Szeged, Hungary \\ Email: knipl@math.u-szeged.hu \\ $\dagger$ Bolyai Institute, University of Szeged, Szeged, Hungary \\ Email: rost@math.u-szeged.hu
}

Received: 12 July 2012, accepted: 25 September 2012, published: 18 October 2012

\begin{abstract}
We present a general epidemic model to describe the spread of an infectious disease in several regions connected by transportation. We take into account that infected individuals not only carry the disease to a new place while traveling from one region to another, but transmit the disease during travel as well. We obtain that a model structured by travel time is equivalent to a large system of differential equations with multiple delays. By showing the local Lipschitz property of the dynamically defined delayed feedback function, we obtain existence and uniqueness of solutions of the system.
\end{abstract}

Keywords-epidemic spread; transportation model; dynamically defined delay; Lipschitz continuity

\section{INTRODUCTION}

We consider an arbitrary $n$ number of regions which are connected by transportation, and present an SIR based model which describes the spread of infection in the regions and also during travel between them. We show that our model is equivalent to a system of functional differential equations

$$
x^{\prime}(t)=\mathcal{F}\left(x_{t}\right),
$$

where $t \in \mathbb{R}, t \geq 0$ and $x: \mathbb{R} \rightarrow \mathbb{R}^{3 n}$. We use the notation $x_{t} \in \mathcal{C}, x_{t}(\theta)=x(t+\theta)$ for $\theta \in[-\sigma, 0]$, where for $\sigma>0$, we define our phase space $\mathcal{C}=\mathcal{C}\left([-\sigma, 0], \mathbb{R}^{3 n}\right)$ as the Banach space of continuous functions from $[-\sigma, 0]$ to $\mathbb{R}^{3 n}$, equipped with the usual supremum norm $\|\cdot\|$. In the sequel we use the notation $|v|$ for the Euclidean
TABLE I

VARIABLES AND KEY MODEL PARAMETERS $(j, k \in\{1, \ldots n\})$

\begin{tabular}{|c||c|}
\hline $\begin{array}{c}S_{j}, I_{j}, \\
R_{j}, N_{j}\end{array}$ & $\begin{array}{c}\text { susceptible, infected, recovered, all } \\
\text { individuals in region } j\end{array}$ \\
\hline $\begin{array}{c}s_{k, j}, i_{k, j}, \\
r_{k, j}, n_{k, j}\end{array}$ & $\begin{array}{c}\text { susceptible, infected, recovered, all } \\
\text { individuals during travel from region } k \text { to } j\end{array}$ \\
\hline$\Lambda_{j}$ & incidence in region $j$ \\
\hline$\lambda_{k, j}$ & incidence during travel from region $k$ to $j$ \\
\hline$\alpha_{j}$ & recovery rate of infected individuals in region $j$ \\
\hline$\alpha_{k, j}$ & recovery rate during travel from region $k$ to $j$ \\
\hline$\mu_{j, k}$ & travel rate from region $j$ to region $k$ \\
\hline$\tau_{k, j}$ & duration of travel from region $k$ to $j$ \\
\hline
\end{tabular}

norm of any vector $v \in \mathbb{R}^{m}$ for $m \in \mathbb{Z}_{+}$. In order to obtain the general existence and uniqueness result for the system, we prove that $\mathcal{F}: \mathcal{C} \rightarrow \mathbb{R}^{3 n}$ satisfies the local Lipschitz condition on each bounded subset of $\mathcal{C}$, that is, for every $M>0$ there exists a constant $K=K(M)$ such that the inequality $|\mathcal{F}(\phi)-\mathcal{F}(\psi)| \leq K|| \phi-\psi \|$ holds for every $\phi, \psi \in \mathcal{C}$ with $\|\phi\|,\|\psi\| \leq M$.

The paper is organised as follows. In Section 2 we introduce our model, then we obtain the compact form of the system in Section 3. Section 4 concerns with the proof of the local Lipschitz condition for various types of incidence (new cases per unit of time). 


\section{Knipl et al., Multiregional SIR Model with Infection during Transportation}

\section{MODEL DESCRIPTION}

We formulate a dynamical model describing the spread of an infectious disease in $n$ regions and also during travel from one region to another. Divide the entire populations of the $n$ regions into the disjoint classes $S_{j}, I_{j}, R_{j}, j \in\{1, \ldots n\}$, where $S_{j}(t) I_{j}(t)$, $R_{j}(t), j \in\{1, \ldots n\}$ denote the number of susceptible, infected and recovered individuals at time $t$ in region $j$. For the total population in region $j$ at time $t$, we use the notation

$$
N_{j}(t)=S_{j}(t)+I_{j}(t)+R_{j}(t)
$$

The incidence in region $j$ is denoted by $\Lambda_{j}\left(S_{j}(t), I_{j}(t), R_{j}(t)\right)$, model parameter $\alpha_{j}$ represents the recovery rate of infected individuals in region $j$. We denote the travel rate from region $j$ to region $k$ by $\mu_{j, k}$ and we set $\mu_{j, j}=0$ for $j, k \in\{1, \ldots n\}$.

Let $s_{k, j}, i_{k, j}, r_{k, j}$ denote susceptible, infected and recovered travelers, where lower index-pair $\{k, j\}$, $j, k \in\{1, \ldots n\}$ indicates that individuals are traveling from region $k$ to region $j$. Let $\tau_{k, j}>0$ denote the time required to complete the travel from region $k$ to region $j$, which is assumed to be fixed. To describe the disease dynamics during travel, for each $t_{*}$ we define $s_{k, j}\left(u ; t_{*}\right), i_{k, j}\left(u ; t_{*}\right), r_{k, j}\left(u ; t_{*}\right), j, k \in\{1, \ldots n\}$ as the densities of individuals with respect to $u$ who started travel at time $t_{*}$ and belong to classes $s_{k, j}, i_{k, j}, r_{k, j}$, where $u \in\left[0, \tau_{k, j}\right]$ denotes the time elapsed since the beginning of the travel. Then $s_{k, j}\left(\tau_{k, j} ; t-\tau_{k, j}\right)$, $i_{k, j}\left(\tau_{k, j} ; t-\tau_{k, j}\right), r_{k, j}\left(\tau_{k, j} ; t-\tau_{k, j}\right)$ express the inflow of individuals arriving from region $k$ to compartments $S_{j}, I_{j}, R_{j}$ at time $t$, respectively. Let

$$
n_{k, j}\left(u ; t_{*}\right)=s_{k, j}\left(u ; t_{*}\right)+i_{k, j}\left(u ; t_{*}\right)+r_{k, j}\left(u ; t_{*}\right)
$$

denote the total density of individuals during travel from region $k$ to $j$, where $j, k \in\{1, \ldots n\}$. The total density is constant during travel, i.e. $n_{k, j}\left(u ; t_{*}\right)=n_{k, j}\left(0, t_{*}\right)$ for all $u \in\left[0, \tau_{k, j}\right]$. During the course of travel from region $k$ to $j, \lambda_{k, j}\left(s_{k, j}\left(u ; t_{*}\right), i_{k, j}\left(u ; t_{*}\right), r_{k, j}\left(u ; t_{*}\right)\right)$ represents the incidence, and let $\alpha_{k, j}$ denote the recovery rate.

All variables and model parameters are listed in Table I Based on the assumptions formulated above, we obtain the following system of differential equations for the disease transmission in region $j, j \in\{1, \ldots n\}$ :

$$
\left\{\begin{aligned}
\dot{S}_{j}(t) & =-\Lambda_{j}(\cdot)-\left(\sum_{k=1}^{n} \mu_{j, k}\right) S_{j}(t) \\
& +\sum_{k=1}^{n} s_{k, j}\left(\tau_{k, j} ; t-\tau_{k, j}\right), \\
\dot{I}_{j}(t) & =\Lambda_{j}(\cdot)-\left(\sum_{k=1}^{n} \mu_{j, k}\right) I_{j}(t) \\
& -\alpha_{j} I_{j}(t)+\sum_{k=1}^{n} i_{k, j}\left(\tau_{k, j} ; t-\tau_{k, j}\right), \\
\dot{R}_{j}(t) & =\alpha_{j} I_{j}(t)-\left(\sum_{k=1}^{n} \mu_{j, k}\right) R_{j}(t) \\
& +\sum_{k=1}^{n} r_{k, j}\left(\tau_{k, j} ; t-\tau_{k, j}\right) .
\end{aligned}\right.
$$

For each $j, k \in\{1, \ldots n\}$ and for each $t_{*}$, the following system $\left(T_{k, j}\right)$ describes the evolution of the densities during the travel from region $k$ to region $j$ which started at time $t_{*}$ :

$$
\left\{\begin{array}{l}
\frac{\mathrm{d}}{\mathrm{d} u} s_{k, j}\left(u ; t_{*}\right)=-\lambda_{k, j}(\cdot), \\
\frac{\mathrm{d}}{\mathrm{d} u} i_{k, j}\left(u ; t_{*}\right)=\lambda_{k, j}(\cdot)-\alpha_{k, j} i_{k, j}\left(u ; t_{*}\right), \\
\frac{\mathrm{d}}{\mathrm{d} u} r_{k, j}\left(u ; t_{*}\right)=\alpha_{k, j} i_{k, j}\left(u ; t_{*}\right) .
\end{array}\right.
$$

For sake of simplicity, in systems $\left(L_{j}\right)$ and $\left(T_{k, j}\right)$ we use the notations $\Lambda_{j}(\cdot)$ and $\lambda_{k, j}(\cdot)$ for the incidences, where these functions are meant to be evaluated at the appropriate points. For $u=0$, the densities are determined by the rates individuals start their travels from region $k$ to region $j$ at time $t_{*}$. Hence, the initial values for system $\left(T_{k, j}\right)$ at $u=0$ are given by

$$
\left\{\begin{aligned}
s_{k, j}\left(0 ; t_{*}\right) & =\mu_{k, j} S_{k}\left(t_{*}\right), \\
i_{k, j}\left(0 ; t_{*}\right) & =\mu_{k, j} I_{k}\left(t_{*}\right), \\
r_{k, j}\left(0 ; t_{*}\right) & =\mu_{k, j} R_{k}\left(t_{*}\right) .
\end{aligned}\right.
$$

Notice that $\mu_{j, j}=0$ for $j \in\{1, \ldots n\}$ implies that for each $t_{*}$, it holds that $s_{j, j}\left(u ; t_{*}\right)=i_{j, j}\left(u ; t_{*}\right)=$ $r_{j, j}\left(u ; t_{*}\right) \equiv 0$, as there is no travel from region $j$ to itself. Since travel from region $k$ to region $j$ takes $\tau_{k, j}$ units of time to complete, we need to assure that there exists a unique solution of system $\left(T_{k, j}\right)$ on $\left[0, \tau_{k, j}\right]$ (see Proposition IV.1.

Now we turn our attention to the terms $s_{k, j}\left(\tau_{k, j} ; t-\tau_{k, j}\right)$, $i_{k, j}\left(\tau_{k, j} ; t-\tau_{k, j}\right), r_{k, j}\left(\tau_{k, j} ; t-\tau_{k, j}\right), j, k \in\{1, \ldots n\}$ in 


\section{Knipl et al., Multiregional SIR Model with Infection during Transportation}

system $\left(L_{j}\right)$, which give the inflow of individuals arriving to classes $S_{j}, I_{j}, R_{j}$ at time $t$, upon completing a travel from region $k$. At time $t$, these terms are determined by the solution of system $\left(T_{k, j}\right)$ at $u=\tau_{k, j}$ with initial values $\left(I V T_{k, j}\right)$ for $t_{*}=t-\tau_{k, j}$, since individuals who left region $k$ with rate $\mu_{k, j}$ at time $t-\tau_{k, j}$ will enter region $j$ at time $t$.

Next we specify initial values for system $\left(L_{j}\right)$ at $t=0$. Since for $k \in\{1, \ldots n\}$, travel from region $k$ to region $j$ takes $\tau_{k, j}$ units of time to complete, arrivals to region $j$ at time $t$ are determined by the state of the classes of region $k$ at $t-\tau_{k, j}$, via the solution of system $\left(T_{k, j}\right)$ and initial values $\left(I V T_{k, j}\right)$. Thus, we set up initial values as follows:

$$
\left\{\begin{aligned}
S_{j}(\theta) & =\varphi_{S, j}(\theta), \\
I_{j}(\theta) & =\varphi_{I, j}(\theta), \\
R_{j}(\theta) & =\varphi_{R, j}(\theta),
\end{aligned}\right.
$$

where $\theta \in[-\tau, 0]$ for $\tau:=\max _{j, k \in\{1, \ldots n\}} \tau_{k, j}$, moreover $\varphi_{S, j}, \varphi_{I, j}$ and $\varphi_{R, j}$ are continuous functions for $j \in$ $\{1, \ldots n\}$.

\section{The Compact Form of the System}

For each $j, k \in\{1, \ldots n\}$ and $t_{*} \geq 0$, we define $y(u)=y_{k, j}^{t_{*}}(u)=\left(s_{k, j}\left(u ; t_{*}\right), i_{k, j}\left(u ; t_{*}\right), r_{k, j}\left(u ; t_{*}\right)\right)^{T}$ and $g=g_{k, j}=\left(g_{S}, g_{I}, g_{R}\right)^{T}$, where $y:\left[0, \tau_{k, j}\right] \rightarrow \mathbb{R}^{3}$, $g: \mathbb{R}^{3} \rightarrow \mathbb{R}^{3}$ and

$$
\begin{aligned}
g_{S}(y) & =-\lambda_{k, j}\left(y_{1}, y_{2}, y_{3}\right), \\
g_{I}(y) & =\lambda_{k, j}\left(y_{1}, y_{2}, y_{3}\right)-\alpha_{k, j} y_{2}, \\
g_{R}(y) & =\alpha_{k, j} y_{2} .
\end{aligned}
$$

Then for each $j, k$ and $t_{*}$, system

$$
\left\{\begin{aligned}
y^{\prime}(u) & =g(y(u)) \\
y(0) & =y_{0}
\end{aligned}\right.
$$

is a compact form of system $\left(T_{k, j}\right)$ with initial values $\left(I V T_{k, j}\right)$ for $y_{0}=\left(\mu_{k, j} S_{k}\left(t_{*}\right), \mu_{k, j} I_{k}\left(t_{*}\right)\right.$, $\left.\mu_{k, j} R_{k}\left(t_{*}\right)\right)^{T}$. Let $y\left(u, 0 ; y_{0}\right)$ denote the solution of system (2) at time $u$ with initial value $y_{0}$.

The feasible phase space is the nonnegative cone $\mathcal{C}_{+}=$ $\mathcal{C}\left([-\tau, 0], \mathbb{R}_{+}^{3 n}\right)$ of the Banach space of continuous functions from $[-\tau, 0]$ to $\mathbb{R}^{3 n}$ with the sup norm. For every $j, k \in\{1, \ldots n\}$, let $h_{k, j}: \mathbb{R}^{3 n} \rightarrow \mathbb{R}^{3}$ be defined by $h_{k, j}=\left(h_{S, k, j}, h_{I, k, j}, h_{R, k, j}\right)^{T}$, where

$$
\begin{aligned}
h_{S, k, j}(v) & =\mu_{k, j} v_{3 k-2}, \\
h_{I, k, j}(v) & =\mu_{k, j} v_{3 k-1}, \\
h_{R, k, j}(v) & =\mu_{k, j} v_{3 k} .
\end{aligned}
$$

For $\phi \in \mathcal{C}_{+}$, we use the notation $\hat{y}_{\phi\left(-\tau_{k, j}\right)}(u)=$ $y\left(u, 0 ; h_{k, j}\left(\phi\left(-\tau_{k, j}\right)\right)\right)$. Furthermore we define $W_{k}$ : $\mathcal{C}_{+} \rightarrow \mathbb{R}^{3 n}$ as

$$
W_{k}(\phi)=\left(\hat{y}_{\phi\left(-\tau_{k, 1}\right)}\left(\tau_{k, 1}\right), \ldots \hat{y}_{\phi\left(-\tau_{k, n}\right)}\left(\tau_{k, n}\right)\right)^{T} .
$$

Let $x(t)=\left(S_{1}(t), I_{1}(t), R_{1}(t), \ldots S_{n}(t), I_{n}(t), R_{n}(t)\right)^{T}$ for $t \geq 0$, and $f=\left(f_{S, 1}, f_{I, 1}, f_{R, 1}, \ldots f_{S, n}, f_{I, n}, f_{R, n}\right)^{T}$, where for $j \in\{1, \ldots n\}$,

$$
\begin{aligned}
f_{S, j}(x) & =-\Lambda_{j}\left(x_{3 j-2}, x_{3 j-1}, x_{3 j}\right)-\left(\sum_{k=1}^{n} \mu_{j, k}\right) x_{3 j-2}, \\
f_{I, j}(x) & =\Lambda_{j}\left(x_{3 j-2}, x_{3 j-1}, x_{3 j}\right)-\alpha_{j} x_{3 j-1} \\
& -\left(\sum_{k=1}^{n} \mu_{j, k}\right) x_{3 j-1} \\
f_{R, j}(x) & =\alpha_{j} x_{3 j-1}-\left(\sum_{k=1}^{n} \mu_{j, k}\right) x_{3 j} .
\end{aligned}
$$

Clearly the union of systems $\left(L_{j}\right)$ with initial conditions $\left(I V L_{j}\right), j \in\{1, \ldots n\}$ can be written in a closed form as

$$
\left\{\begin{aligned}
x^{\prime}(t) & =f(x(t))+\sum_{k=1}^{n} W_{k}\left(x_{t}\right)=: \mathcal{F}\left(x_{t}\right), \\
x_{0} & =\varphi
\end{aligned}\right.
$$

where $\mathcal{F}: \mathcal{C}_{+} \rightarrow \mathbb{R}^{3 n}$ and for $\varphi \in \mathcal{C}_{+}, \varphi:=$ $\left(\varphi_{S, 1}, \varphi_{I, 1}, \varphi_{R, 1}, \ldots, \varphi_{S, n}, \varphi_{I, n}, \varphi_{R, n}\right)^{T}$.

\section{THE LOCAL LipsChitz PROPERTY}

This section is devoted to the proof of the general existence and uniqueness result of system (3). First, we obtain the following simple result.

Proposition IV.1. Assume that $\lambda_{k, j}$ possesses the local Lipschitz property on each bounded subset of $\mathbb{R}^{3}$. Moreover, assume that $\lambda_{k, j}\left(q_{1}, q_{2}, q_{3}\right) \geq 0$ and $\lambda_{k, j}\left(0, q_{2}, q_{3}\right)=0$ hold for $q_{1}, q_{2}, q_{3} \geq 0$. Then there exists a unique solution of system (2) which continuously depends on the initial data, and for $u \in[0, \tau]$ and $y_{0} \geq 0$ the following inequality holds componentwise:

$$
0 \leq y\left(u, 0 ; y_{0}\right) \leq \sqrt{3}\left|y_{0}\right| .
$$

Proof: The local Lipschitz condition guarantees the existence of a unique solution which continuously depends on the initial data (see Picard-Lindelöf theorem in Chapter II, Theorem 1.1 and Chapter V, Theorem 2.1 in [1]). We have also seen that $n_{k, j}\left(u ; t_{*}\right)$ is constant for all $u$ in the maximal interval of existence, moreover from the nonnegativity condition of $\lambda_{k, j}$ it follows that 
nonnegative initial data give rise to nonnegative solution. Hence we obtain

$$
\begin{aligned}
0 & \leq n_{k, j}\left(0 ; t_{*}\right)=n_{k, j}\left(u ; t_{*}\right), \\
0 & \leq s_{k, j}\left(0 ; t_{*}\right)+i_{k, j}\left(0 ; t_{*}\right)+r_{k, j}\left(0 ; t_{*}\right) \\
& =s_{k, j}\left(u ; t_{*}\right)+i_{k, j}\left(u ; t_{*}\right)+r_{k, j}\left(u ; t_{*}\right) \\
& =\mu_{k, j}\left(S_{k}\left(t_{*}\right)+I_{k}\left(t_{*}\right)+R_{k}\left(t_{*}\right)\right), \\
0 & \leq s_{k, j}\left(u ; t_{*}\right), i_{k, j}\left(u ; t_{*}\right), r_{k, j}\left(u ; t_{*}\right) \\
& \leq \mu_{k, j}\left(S_{k}\left(t_{*}\right)+I_{k}\left(t_{*}\right)+R_{k}\left(t_{*}\right)\right),
\end{aligned}
$$

where we used $\left(I V T_{k, j}\right)$. Using the definition of $y$, (4) implies that the inequality

$$
\begin{aligned}
0 & \leq\left(y\left(u, 0 ; y_{0}\right)\right)_{1},\left(y\left(u, 0 ; y_{0}\right)\right)_{2},\left(y\left(u, 0 ; y_{0}\right)\right)_{3} \\
& \leq\left(y_{0}\right)_{1}+\left(y_{0}\right)_{2}+\left(y_{0}\right)_{3} \\
& \leq \sqrt{3} \sqrt{\left(\left(y_{0}\right)_{1}\right)^{2}+\left(\left(y_{0}\right)_{2}\right)^{2}+\left(\left(y_{0}\right)_{3}\right)^{2}}
\end{aligned}
$$

holds for $u \in[0, \infty)$, where $y_{0}=\left(\left(y_{0}\right)_{1},\left(y_{0}\right)_{2},\left(y_{0}\right)_{3}\right)^{T}$ is the initial value and we used the arithmetic-quadratic mean inequality. We conclude that the solution exists on $[0, \tau]$ and is bounded.

Now we prove that if we assume that $\Lambda_{j}$ and $\lambda_{k, j}$ possess the local Lipschitz property, then $\mathcal{F}$ is also locally Lipschitz continuous.

Lemma IV.2. Let us suppose that for all $j, k \in$ $\{1, \ldots n\}, \Lambda_{j}$ and $\lambda_{k, j}$ possess the local Lipschitz property, $\lambda_{k, j}\left(q_{1}, q_{2}, q_{3}\right) \geq 0$ and $\lambda_{k, j}\left(0, q_{2}, q_{3}\right)=0$ hold for $q_{1}, q_{2}, q_{3} \geq 0$. Then $\mathcal{F}$ satisfies the local Lipschitz condition on each bounded subset of $\mathcal{C}_{+}$.

Proof: We claim that for every $M>0$ there exists a constant $K=K(M)$ such that the inequality $|\mathcal{F}(\phi)-\mathcal{F}(\psi)| \leq K\|\phi-\psi\|$ holds for every $\phi, \psi \in \mathcal{C}_{+}$ with $\|\phi\|,\|\psi\| \leq M$.

Fix indices $j, k \in\{1, \ldots n\}$. For $\|\psi\| \leq M$ it holds componentwise that $0 \leq \psi\left(-\tau_{k, j}\right) \leq M$, so due to the continuity of $h_{k, j}$, there exists a constant $L_{k, j}^{h}(M)$ such that $0 \leq h_{k, j}\left(\psi\left(-\tau_{k, j}\right)\right) \leq L_{k, j}^{h}$ is satisfied componentwise. For $y_{0}=h_{k, j}\left(\psi\left(-\tau_{k, j}\right)\right)$ Proposition IV.1 implies that there exists a $J_{k, j}=J_{k, j}\left(L_{k, j}^{h}\right)=J_{k, j}(M)$ such that the inequality $\left|\hat{y}_{\psi\left(-\tau_{k, j}\right)}(u)\right| \leq J_{k, j}$ holds for $u \in[0, \tau]$ (for instance we can choose $J_{k, j}=\sqrt{3} L_{k, j}^{h}$ ).

The local Lipschitz property of $h_{k, j}$ follows from its definition. We assumed that $\lambda_{k, j}$ is Lipschitz continuous, this implies the Lipschitz continuity of $g$. Let $K_{k, j}^{h}=K_{k, j}^{h}(M)$ be the Lipschitz constant of $h_{k, j}$ on the set $\left\{v \in \mathbb{R}^{3 n}:|v| \leq M\right\}$, we denote the Lipschitz constant of $g=g_{k, j}$ on the set $\left\{v \in \mathbb{R}^{3}:|v| \leq J_{k, j}\right\}$ by $K_{k, j}^{g}=K_{k, j}^{g}(J)=K_{k, j}^{g}(M)$. For any $\|\phi\|,\|\psi\| \leq M$, it holds that $\left|\phi\left(-\tau_{k, j}\right)\right|,\left|\psi\left(-\tau_{k, j}\right)\right| \leq M$. Since solutions of (2) can be expressed as $y\left(u, 0 ; y_{0}\right)=y_{0}+$ $\int_{0}^{u} g\left(y\left(r, 0 ; y_{0}\right)\right) \mathrm{d} r$, we have

$$
\begin{aligned}
& \left|\hat{y}_{\phi\left(-\tau_{k, j}\right)}(u)-\hat{y}_{\psi\left(-\tau_{k, j}\right)}(u)\right| \\
& =\mid h_{k, j}\left(\phi\left(-\tau_{k, j}\right)\right)+\int_{0}^{u} g\left(\hat{y}_{\phi\left(-\tau_{k, j}\right)}(r)\right) \mathrm{d} r \\
& -\left(h_{k, j}\left(\psi\left(-\tau_{k, j}\right)\right)+\int_{0}^{u} g\left(\hat{y}_{\psi\left(-\tau_{k, j}\right)}(r)\right) \mathrm{d} r\right) \mid \\
& \leq\left|h_{k, j}\left(\phi\left(-\tau_{k, j}\right)\right)-h_{k, j}\left(\psi\left(-\tau_{k, j}\right)\right)\right| \\
& +\int_{0}^{u}\left|g\left(\hat{y}_{\phi\left(-\tau_{k, j}\right)}(r)\right)-g\left(\hat{y}_{\psi\left(-\tau_{k, j}\right)}(r)\right)\right| \mathrm{d} r \\
& \leq K_{k, j}^{h}|| \phi-\psi|| \\
& +\int_{0}^{u} K_{k, j}^{g}\left|\hat{y}_{\phi\left(-\tau_{k, j}\right)}(r)-\hat{y}_{\psi\left(-\tau_{k, j}\right)}(r)\right| \mathrm{d} r
\end{aligned}
$$

for $u \in[0, \tau]$. Define

$$
\Gamma(u)=\left|\hat{y}_{\phi\left(-\tau_{k, j}\right)}(u)-\hat{y}_{\psi\left(-\tau_{k, j}\right)}(u)\right|
$$

for $u \in[0, \tau]$. Then $(5)$ gives

$$
\Gamma(u) \leq K_{k, j}^{h}\|\phi-\psi\|+K_{k, j}^{g} \int_{0}^{u} \Gamma(r) \mathrm{d} r,
$$

and from Gronwall's inequality we have

$$
\Gamma(u) \leq K_{k, j}^{h}\|\phi-\psi\| e^{K_{k, j}^{g} u} .
$$

Applying the definition of $W_{k}$, we arrive to the inequality

$$
\begin{aligned}
& \left|\left(W_{k}(\phi)\right)_{j}-\left(W_{k}(\psi)\right)_{j}\right| \\
& =\left|\hat{y}_{\phi\left(-\tau_{k, j}\right)}\left(\tau_{k, j}\right)-\hat{y}_{\psi\left(-\tau_{k, j}\right)}\left(\tau_{k, j}\right)\right| \\
& \leq K_{k, j}^{h} e^{K_{k, j}^{g} \tau_{k, j}}|| \phi-\psi \|,
\end{aligned}
$$

where we used (6) at $u=\tau_{k, j}$.

It is straightforward that $W_{k}$ has the Lipschitz condition for any $k \in\{1, \ldots n\}, K^{W_{k}}=K^{W_{k}}(M)=$ $\sqrt{\sum_{j=1}^{n}\left(K_{k, j}^{h} e^{K_{k, j}^{g} \tau_{k, j}}\right)^{2}}$ is a suitable choice for the Lipschitz constant.

Finally, the assumption that $\Lambda_{j}$ is Lipschitz continuous for any $j \in\{1, \ldots n\}$ implies the Lipschitz continuity of $f$, so let $K^{f}=K^{f}(M)$ be the Lipschitz constant of $f$ on the set $\left\{v \in \mathbb{R}^{3 n}:|v| \leq M\right\}$. Then for any ||$\phi||,|| \psi \| \leq M,|\phi(0)|,|\psi(0)|,|\phi(-\tau)|,|\psi(-\tau)| \leq M$ hold and thus

$$
\begin{aligned}
|\mathcal{F}(\phi)-\mathcal{F}(\psi)| & \leq|f(\phi(0))-f(\psi(0))| \\
& +\sum_{k=1}^{n}\left|W_{k}(\phi)-W_{k}(\psi)\right| \\
& \leq K^{f}|| \phi-\psi\left\|+\sum_{k=1}^{n} K^{W_{k}}|| \phi-\psi\right\| .
\end{aligned}
$$


D. Knipl et al., Multiregional SIR Model with Infection during Transportation

$$
\begin{aligned}
\left|\Lambda_{1}(\mathbf{p})-\Lambda_{1}(\mathbf{q})\right| & =\beta_{1}\left|\frac{q_{1} q_{2}}{q_{1}+q_{2}+q_{3}}-\frac{p_{1} p_{2}}{p_{1}+p_{2}+p_{3}}\right| \\
& =\beta_{1} \mid \frac{q_{1} q_{2}}{q_{1}+q_{2}+q_{3}}-\frac{q_{1} p_{2}}{q_{1}+q_{2}+q_{3}}+\frac{q_{1} p_{2}}{q_{1}+q_{2}+q_{3}} \\
& -\frac{q_{1} p_{2}}{q_{1}+p_{2}+q_{3}}+\frac{q_{1} p_{2}}{q_{1}+p_{2}+q_{3}}-\frac{q_{1} p_{2}}{q_{1}+p_{2}+p_{3}} \\
& +\frac{q_{1} p_{2}}{q_{1}+p_{2}+p_{3}}-\frac{q_{1} p_{2}}{p_{1}+p_{2}+p_{3}}+\frac{q_{1} p_{2}}{p_{1}+p_{2}+p_{3}}-\frac{p_{1} p_{2}}{p_{1}+p_{2}+p_{3}} \mid \\
& \leq \beta_{1}\left(\left|\frac{q_{1} q_{2}}{q_{1}+q_{2}+q_{3}}-\frac{q_{1} p_{2}}{q_{1}+q_{2}+q_{3}}\right|\right. \\
& +\left|\frac{q_{1} p_{2}}{q_{1}+q_{2}+q_{3}}-\frac{q_{1} p_{2}}{q_{1}+p_{2}+q_{3}}\right|+\left|\frac{q_{1} p_{2}}{q_{1}+p_{2}+q_{3}}-\frac{q_{1} p_{2}}{q_{1}+p_{2}+p_{3}}\right| \\
& \left.+\left|\frac{q_{1} p_{2}}{q_{1}+p_{2}+p_{3}}-\frac{q_{1} p_{2}}{p_{1}+p_{2}+p_{3}}\right|+\left|\frac{q_{1} p_{2}}{p_{1}+p_{2}+p_{3}}-\frac{p_{1} p_{2}}{p_{1}+p_{2}+p_{3}}\right|\right) \\
& =\beta_{1}\left(\left|q_{2}-p_{2}\right|\left|\frac{q_{1}}{q_{1}+q_{2}+q_{3}}\right|+\left|p_{2}-q_{2}\right|\left|\frac{q_{1} p_{2}}{\left(q_{1}+q_{2}+q_{3}\right)\left(q_{1}+p_{2}+q_{3}\right)}\right|\right. \\
& +\left|p_{3}-q_{3}\right|\left|\frac{q_{1} p_{2}}{\left(q_{1}+p_{2}+q_{3}\right)\left(q_{1}+p_{2}+p_{3}\right)}\right| \\
& \left.+\left|p_{1}-q_{1}\right|\left|\frac{q_{1} p_{2}}{\left(q_{1}+p_{2}+p_{3}\right)\left(p_{1}+p_{2}+p_{3}\right)}\right|+\left|q_{1}-p_{1}\right|\left|\frac{p_{2}}{p_{1}+p_{2}+p_{3}}\right|\right) \\
& \leq \beta_{1}\left(2\left|q_{2}-p_{2}\right|+\left|p_{3}-q_{3}\right|+2\left|p_{1}-q_{1}\right|\right) \\
& \leq 5 \beta_{1}|\mathbf{q}-\mathbf{p}|
\end{aligned}
$$

Hence $K^{f}+\sum_{k=1}^{n} \sqrt{\sum_{j=1}^{n}\left(K_{k, j}^{h} e^{K_{k, j}^{g} \tau_{k, j}}\right)^{2}}$ is a suitable choice for $K$, the Lipschitz constant of $\mathcal{F}$ for the set $\left\{\psi \in \mathcal{C}_{+}:\|\psi\| \leq M\right\}$.

The assumptions of Lemma IV.2 on the incidences $\Lambda_{j}\left(S_{j}(t), I_{j}(t), R_{j}(t)\right)$ and $\lambda_{k, j}\left(s_{k, j}\left(u ; t_{*}\right), i_{k, j}\left(u ; t_{*}\right), r_{k, j}\left(u ; t_{*}\right)\right)$ can be fulfilled by several choices on the type of disease transmission. For instance, let $\beta_{j}>0$ be the transmission rate in region $j$ and let $\beta_{k, j}^{T}>0$ denote the transmission rates during travel. For $j, k \in\{1, \ldots n\}$ and for $\mathbf{q}=\left(q_{1}, q_{2}, q_{3}\right) \in \mathbb{R}^{3}$, define

$$
\begin{aligned}
\Lambda_{j}(\mathbf{q}) & =-\beta_{j} \frac{q_{1}}{q_{1}+q_{2}+q_{3}} q_{2}, \\
\lambda_{k, j}(\mathbf{q}) & =-\beta_{k, j}^{T} \frac{q_{1}}{q_{1}+q_{2}+q_{3}} q_{2} .
\end{aligned}
$$

This implies that $\Lambda_{j}$ and $\lambda_{k, j}$ have the form

$$
\begin{aligned}
\Lambda_{j}\left(S_{j}, I_{j}, R_{j}\right) & =-\beta_{j} \frac{S_{j}}{N_{j}} I_{j}, \\
\lambda_{k, j}\left(s_{k, j}, i_{k, j}, r_{k, j}\right) & =-\beta_{k, j}^{T} \frac{s_{k, j}}{n_{k, j}} i_{k, j},
\end{aligned}
$$

which is called standard incidence. Now we prove the following existence-uniqueness theorem.
Theorem IV.3. With the incidences $\Lambda_{j}$ and $\lambda_{k, j}$ defined in (8), there exists a unique solution of system (3).

Proof: Recall Theorem 3.7 from [2]:

Suppose that $\mathcal{F}$ satisfies the local Lipschitz property on each bounded subset of $\mathcal{C}_{+}=\mathcal{C}_{+}\left([-\tau, 0], \mathbb{R}_{+}^{3 n}\right)$, moreover let $M>0$. There exists $A>0$, depending only on $M$ such that if $\phi \in \mathcal{C}_{+}$satisfies $\|\phi\| \leq M$, then there exists a unique solution $x(t)=\hat{x}(t, 0 ; \phi)$ of (3), defined on $[-\tau, A]$. In addition, if $K$ is the Lipschitz constant for $\mathcal{F}$ corresponding to $M$, then

$$
\max _{-\tau \leq \eta \leq A}|x(\eta, 0 ; \phi)-x(\eta, 0 ; \psi)| \leq\|\phi-\psi\| e^{K A}
$$

holds for $\|\phi\|,\|\psi\| \leq M$.

We showed in Lemma IV.2 that the local Lipschitz continuity of $\mathcal{F}$ follows from the local Lipschitz property of the incidences and the nonnegativity condition of $\lambda_{k, j}$. The latter condition clearly holds, hence it is sufficient to prove that the incidences defined in (8) possess the local Lipschitz property.

As one may observe, the definition of the $\Lambda_{j^{-}}$s and $\lambda_{k, j^{-}}$ 
$\mathrm{s}(j, k \in\{1, \ldots n\})$ only differ in a constant multiplier, hence it is sufficent to prove the local Lipschitz property only for one of them, i.e. for $\Lambda_{1}$. Moreover, we prove this property only on the nonnegative cone $\mathbb{R}_{+}^{3}$, which is invariant under systems $(3)$ and $(2)$. For $\mathbf{p}, \mathbf{q} \in \mathbb{R}_{+}^{3}$, by (7) we obtain the Lipschitz constant $K=5 \beta_{1}$, where we used that for any $a, b, c>0$, it holds that $\frac{a}{a+b+c}<1$.

Remark 1. It follows from the proof of Theorem IV.3 that the incidences $\Lambda_{j}$ and $\lambda_{k, j}$ defined in (8) also satisfy the global Lipschitz property, meaning that the Lipschitz constant $K$ arises independently of $M$. In this case, the solution of system (3) exists on $[0, \infty)$.

Another natural choice for the incidences can be the following: for $\mathbf{q}=\left(q_{1}, q_{2}, q_{3}\right) \in \mathbb{R}^{3}$ and for $j, k \in$ $\{1, \ldots n\}$, let

$$
\begin{aligned}
\Lambda_{j}(\mathbf{q}) & =-\beta_{j} q_{1} q_{2}, \\
\lambda_{k, j}(\mathbf{q}) & =-\beta_{k, j}^{T} q_{1} q_{2},
\end{aligned}
$$

which leads to the mass action-type disease transmission, therefore $\Lambda_{j}$ and $\lambda_{k, j}$ have the form

$$
\begin{aligned}
\Lambda_{j}\left(S_{j}, I_{j}, R_{j}\right) & =-\beta_{j} S_{j} I_{j}, \\
\lambda_{k, j}\left(s_{k, j}, i_{k, j}, r_{k, j}\right) & =-\beta_{k, j}^{T} s_{k, j} i_{k, j} .
\end{aligned}
$$

Theorem IV.4. With incidences $\Lambda_{j}$ and $\lambda_{k, j}$ defined in (9), there exists a unique solution of system (3).

Proof: Similarly as by Theorem IV.3, it is enough to show that $\Lambda_{j}$ and $\lambda_{k, j}$ satisfy the local Lipschitz property, we detail the proof only for $\Lambda_{1}$ and consider the nonnegative subspace $R_{+}^{3}$. For any $M>0$, for any $\mathbf{p}, \mathbf{q} \in \mathbb{R}_{+}^{3}$ such that $|\mathbf{p}|,|\mathbf{q}| \leq M$, we obtain

$$
\begin{aligned}
\left|\Lambda_{1}(\mathbf{p})-\Lambda_{1}(\mathbf{q})\right| & =\left|-\beta_{1} p_{1} p_{2}+\beta_{1} q_{1} q_{2}\right| \\
& \leq \beta_{1}\left|p_{1} p_{2}-q_{1} q_{2}\right| \\
& \leq \beta_{1}\left|p_{1} p_{2}-p_{1} q_{2}+p_{1} q_{2}-q_{1} q_{2}\right| \\
& \leq \beta_{1}\left(\left|p_{1} p_{2}-p_{1} q_{2}\right|+\left|p_{1} q_{2}-q_{1} q_{2}\right|\right) \\
& \leq \beta_{1}\left(p_{1}\left|p_{2}-q_{2}\right|+q_{2}\left|p_{1}-q_{1}\right|\right) \\
& \leq 2 M \beta_{1}|\mathbf{q}-\mathbf{p}|,
\end{aligned}
$$

so we can choose $K(M)=2 M \beta_{1}$.

Remark 2. Although the global Lipschitz property does not hold for $\Lambda_{j}$ and $\lambda_{k, j}$ defined in (9), it is possible to show that the solution of (3) is bounded and hence exists on $[0, \infty)$.

\section{CONCLUSION}

The topic of epidemic spread of infectious diseases via transportation networks has recently been examined in several studies (see [3], [4], [5], [6], [7]), although these works mostly consider only two connected regions. We introduced a dynamic model which describes the spread of an infectious disease in and between $n$ regions which are connected by transportation. We used the commonly applied SIR model as a basic epidemic building block in the regions and also during the travel. The model formulation led to a system structured by travel time, which turned out to be equivalent to a system of differential equations with multiple dynamically defined delays. We showed that under local Lipschitz conditions on the infection terms within the regions and during travel, the usual existence and uniqueness results hold.

Recent epidemics like the 2002-2003 SARS outbreak and the 2009 pandemic influenza A(H1N1) highlighted the importance of the global air travel network in the study of epidemic spread. During long distance travel such as intercontinental flights, a single infected individual may infect several other passengers during the flight, and since the progress of these diseases is fast, even a short delay (a fraction of a day) arising due to transportation may play a significant role in the disease dynamics. In this paper we illustrated by proving an existence and uniqueness result that such epidemiological situations can be studied in the framework of delay differential equations.

\section{ACKNOWLEDGMENT}

DHK was partially supported by the Hungarian Research Fund grant OTKA K75517 and the TÁMOP4.2.2/B-10/1-2010-0012 program of the Hungarian National Development Agency. RG was supported by European Research Council StG Nr. 259559, Hungarian Research Fund grant OTKA K75517 and the Bolyai Research Scholarship of Hungarian Academy of Sciences.

\section{REFERENCES}

[1] P. Hartman, "Ordinary differential equations", Classics in Applied Mathematics, vol. 38, SIAM, 2002.

[2] H. L. Smith, An Introduction to Delay Differential Equations with Applications to the Life Sciences, Springer, 2010.

[3] D. H. Knipl, "Fundamental properties of differential equation with dynamically defined delayed feedback", preprint, 2012.

[4] D. H. Knipl, G. Röst, J. Wu,"Epidemic spread of infectious diseases on long distance travel networks", preprint, 2012.

[5] J. Liu, J. Wu, Y. Zhou, "Modeling disease spread via transportrelated infection by a delay differential equation", Rocky Mountain J. Math., vol. 38 No 5, pp. 1225-1541, 2008. 
D. Knipl et al., Multiregional SIR Model with Infection during Transportation

[6] Y. Nakata, "On the global stability of a delayed epidemic model with transport-related infection", Nonlinear Analysis Series B: Real World Applications, vol. 12 No 6, pp. 3028-3034 2011. http://dx.doi.org/10.1016/j.nonrwa.2011.05.004
[7] Y. Nakata, G. Röst, "Global analysis for spread of an infectious disease via global transportation”, submitted, 2012. 